\title{
Management of Complex Pelvic Fracture with Associated Both Column Acetabular Fracture Judet-Letournel Type and a Coccygeal Fracture in a Severe Polytrauma Patient
}

\author{
Jessica Fiolin ${ }^{a}$ Ludwig Andre Powantia Pontoh ${ }^{a, b}$ \\ Ismail Hadisoebroto Dilogo ${ }^{c}$ \\ aJakarta Knee, Shoulder and Orthopaedic Sports Center, Pondok Indah General Hospital, \\ Jakarta, Indonesia; ${ }^{b}$ Department of Orthopaedic and Traumatology, Faculty of Medicine \\ Universitas Indonesia, Fatmawati General Hospital, Jakarta, Indonesia; 'Department of \\ Orthopaedic and Traumatology, Faculty of Medicine Universitas Indonesia, Dr. Cipto \\ Mangunkusumo National Central General Hospital, Jakarta, Indonesia
}

\section{Key Messages}

- Combination of pelvic-acetabular and coccygeal fracture most of the time is accompanied with a severe polytrauma case. We propose a general rule of thumb upon fixation technique in this complicated case of posterior then anterior and outer then inner fixation first. However, besides proper preoperative planning and meticulous surgery intraoperative have been employed, wound complication, prolonged rehabilitation, possibilities of second surgery, and increased length of stay must be anticipated and prepared to ensure a good outcome at the end of the day.

Keywords

Polytrauma - Associated both column acetabular fracture $\cdot$ Pelvic fracture $\cdot$ Coccygeal fracture Case report

\section{Abstract}

Comprehensive emergency managements and early stabilization are pivotal upon treating complex pelvic and acetabular fractures. A thorough operative strategy is required to determine the best operative approach based on the patient's general condition, available facilities, and surgeon preferences in such complex fracture configuration. Advanced technique of the fixation is necessary during a skillful execution of surgery in order to achieve good treatment results. An 18-years-old female crushed by a bus upon crossing street, presented with hypovolemic shock with ISS polytrauma score 50 consisting of right acetabular associated both column fracture, bilateral pelvic fracture anteroposterior compression type 3 , and coccygeal 
fracture with bilateral drop foot. She underwent emergency laparotomy, had her ovary, bladder, and intestine primarily sutured, and then we immobilized the pelvic using anterior frame external fixator, which was maintained for 6 days. Upon stable condition, we performed right ilioinguinal approach and modified Stoppa with lateral window for the left side, while KocherLangenbeck technique was used to approach the posterior acetabular column. Postoperative radiology showed an adequate internal fixation in both right acetabular columns, successful reconstruction of pelvic ring which was fixated the left ischium, left superior and inferior pubic rami, and full restoration of left sacroiliac joint disruption. Majeed pelvic outcome score was 54, while Hannover pelvic outcome score was good and the patient was able to sit without pain 2 months postoperative. Management of complex pelvic-acetabular-coccygeal fracture requires a holistic chain of treatment by emphasizing the prompt emergency management, accurate preoperative planning, and excellent execution of reconstructive surgical strategy to achieve satisfactory outcome.

(C) 2021 The Author(s).

Published by S. Karger AG, Basel

\section{Introduction}

Management of a complex pelvic fracture with associated both column acetabular fracture and a rare coccygeal fracture is challenging. The poor critical condition of patient upon admission requires multidisciplinary approach at the initial management. A severely highenergy injury is regarded in such intriguing case rendering careful total treatment to all body system regions that are involved; meanwhile, immediate orthopedic stabilization should be placed to prevent further deteriorating condition [1].

Management of bilateral pelvic fracture and associated both acetabular columns itself requires a detailed preoperative planning, adequate surgery facilities, and an experienced surgeon. The aim of every treatment concepts in acetabular fractures is an anatomical joint restoration and stable pelvis to maximize function and prevent, or at least delay, the onset of arthritis. Clinical outcomes and the onset of post-traumatic arthritis have been shown to correlate with the accuracy of articular reduction [2-4]. However, patient's medical condition (associated injuries, comorbidities, etc.), physiological age, and functional needs are also key determinants of the treatment algorithm. Most of the time, due to its highenergy mechanism of injury rendering poor patient's condition, this concept is difficult to achieve. In order to accomplish, this congruous reduction, we choose the most suitable approach, which sometimes in such complex case requires more than a single approach. We reported a step-by-step management of a bilateral pelvic fracture and complex acetabular fracture with a coccygeal fracture in a polytrauma patient case underwent surgical management with good outcome.

\section{Case History}

This study had followed the CARE statement of case report criteria and was conducted in accordance with the Declaration of Helsinki and was approved by the local Ethics Committee of the institute. Informed written consent was obtained from the patient prior to their enrollment in this study. Female, 18-years-old unconscious patient who was rolled over by a bus admitted to our emergency department with severe hypovolemic hemorrhagic shock. A simultaneous fluid early and blood resuscitation were performed along with a hemoglobin level of $5.5 \mathrm{~g} / \mathrm{dL}$ and suspicion of ongoing bleeding. Physical

\section{Karger'}


Fig. 1. Pelvic AP radiograph showing bilateral pelvic fracture Marvin Tile C3, right associated both column fracture and coccygeal fracture.
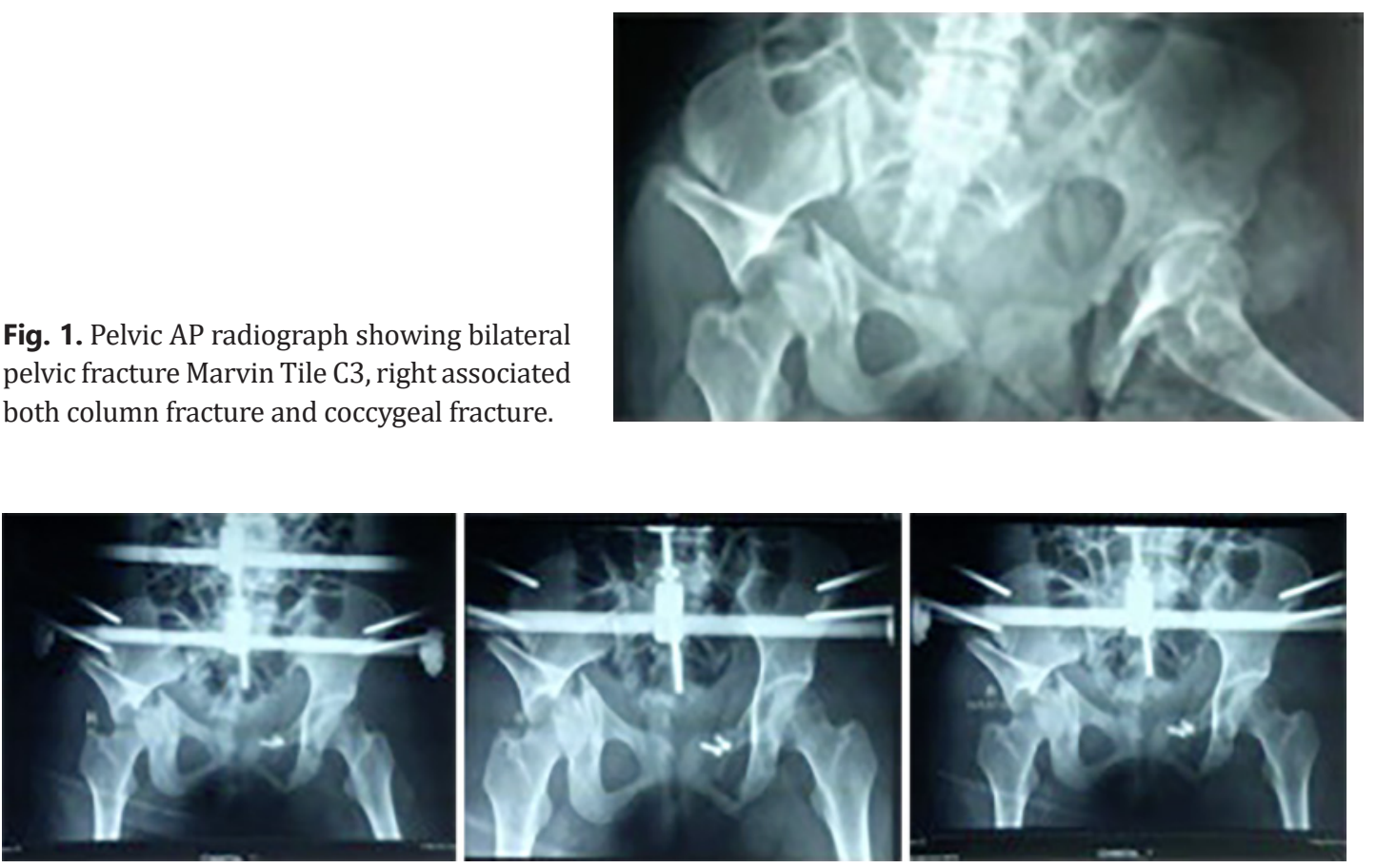

Fig. 2. Pelvic AP, inlet, and outlet radiograph after anterior frame application showing slightly configured pelvic ring congruity.

examination revealed hematoma on her right lower abdomen and bilateral plantar-flexed foot suggesting a pelvic-acetabular fracture with suspicion of sciatic nerve impingement. On the other hand, there were a tense abdomen and hematuria which lead to the suspicion of intra-abdominal and urinal tract injury which then consulted to the general surgery department.

Pelvic radiograph series were ordered promptly showing bilateral pelvic fracture Marvin Tile C3 (Young-Burgess anterior-posterior compression 3), right associated both column and coccygeal fractures (Fig. 1). A Focused Assessment with Sonography in Trauma (FAST) scan was also performed simultaneously showing possibility of gastrointestinal and bladder perforation. Early fluid and blood resuscitation showing nonresponse and signs of ongoing bleeding, patient was assessed with polytrauma Injury Severity Score (ISS) 55 and prepared for emergency laparotomy and anterior frame external fixation.

Intraoperatively, there was an intraperitoneal bladder rupture for about $10 \mathrm{~cm} ; 3 \mathrm{~cm}$ subserous intestine laceration and $3 \mathrm{~cm}$ right ovary rupture which were repaired right away. Anterior frame external fixation was placed to stabilize the pelvic-acetabular complex. Afterward, patient was moved to the intensive care unit, and pelvic AP, inlet, and outlet radiograph along with 3-dimensional reconstruction pelvic computed tomography scan was performed 2 days afterward when the patient hemodynamic was stable to evaluate the fragments of fracture (Fig. 2, 3) showing a pelvic fracture M.

Six days after anterior frame fixation, patient was alert, with stable hemodynamic and normal peripheral blood count. We decided to do definitive surgery by open reduction internal fixation for pelvic-acetabular fracture using plate and screw to achieve anatomic reduction and stable fixation. After a careful planning, we decided to approach the right hip using right ilioinguinal approach in supine position to fixate right iliac wing, right sacroiliac joint, right pubic rami, and right anterior wall and approach the left hemipelvic using modified Stoppa and third left lateral window to fixate the left iliac wing, left sacroiliac joint, left pubic 
Fig. 3. Three-dimensional reconstruction pelvic CT Scan showing fragments of acetabular fracture. CT, computed tomography.

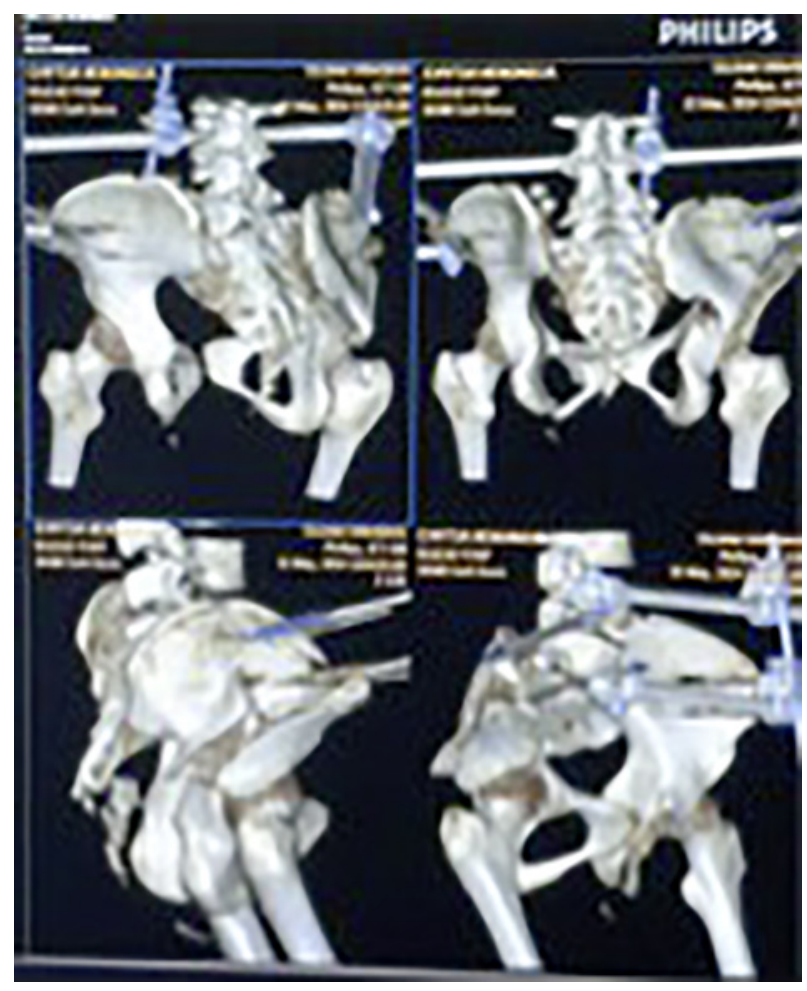

Fig. 4. Complex pelvic-acetabular fracture fixation preoperative planning. Blue line is fixation to the right iliac wing, sacroiliac joint, right pubic rami, and left anterior wall using ilioinguinal approach; yellow line is fixation of left iliac wing, left sacroiliac joint, left pubic rami using modified Stoppa, and left lateral window; and red line indicates the fixation of posterior column using Kocher-Langenbeck.

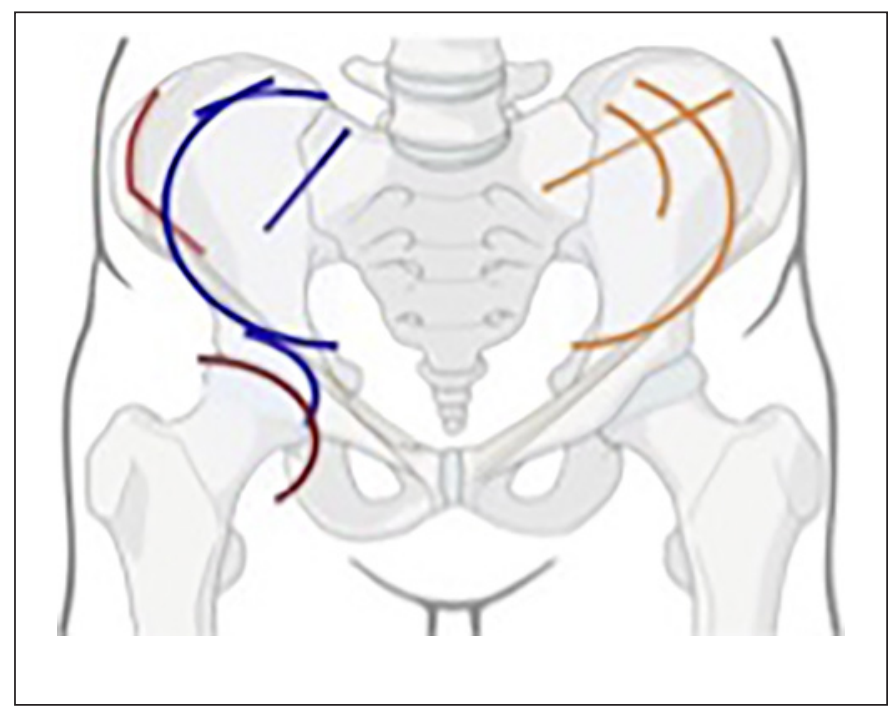

rami, and finally approach the posterior column in lateral decubitus position using KocherLangenbeck approach while leaving the undisplaced coccygeal fracture alone (Fig. 4). Pelvic radiographs were taken afterward to evaluate the pelvic ring, acetabular, and coccygeal fracture fragments (Fig. 5).

Postoperative was performed to maintain hip and knee range of motion, while passive ankle dorsiflexion and active plantar flexion exercise were performed for drop foot therapy. The patient was mobilized using a wheelchair while leaning to right and left was performed every $2 \mathrm{~h}$. 


\section{Case Reports in Orthopedic Research}
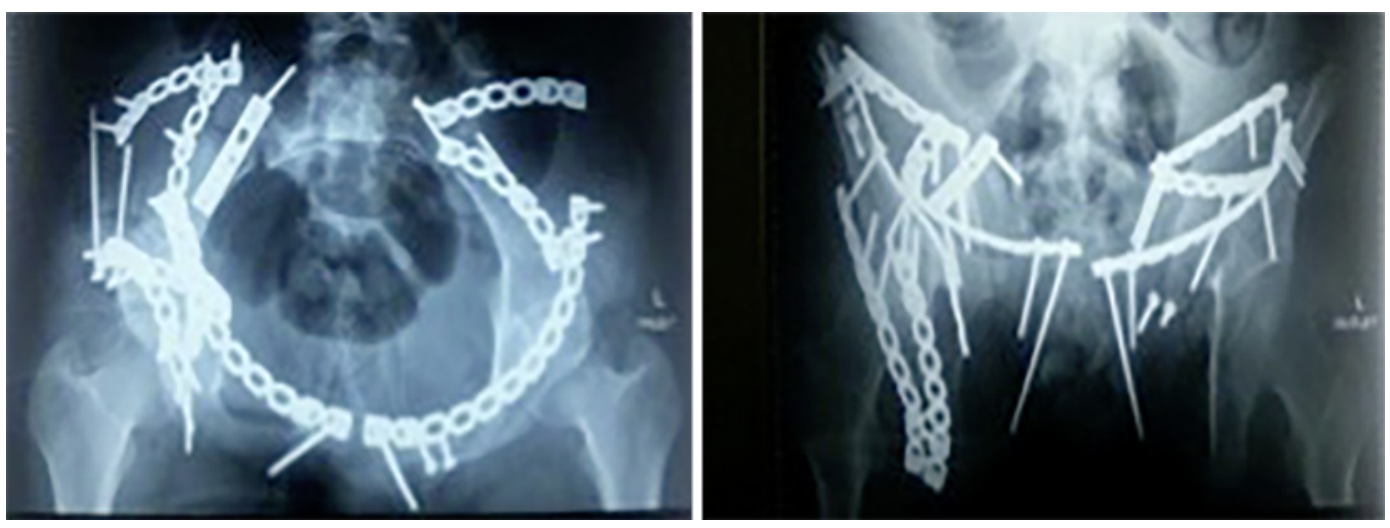

Fig. 5. Final radiograph of open reduction internal fixation pelvic-acetabular complex.
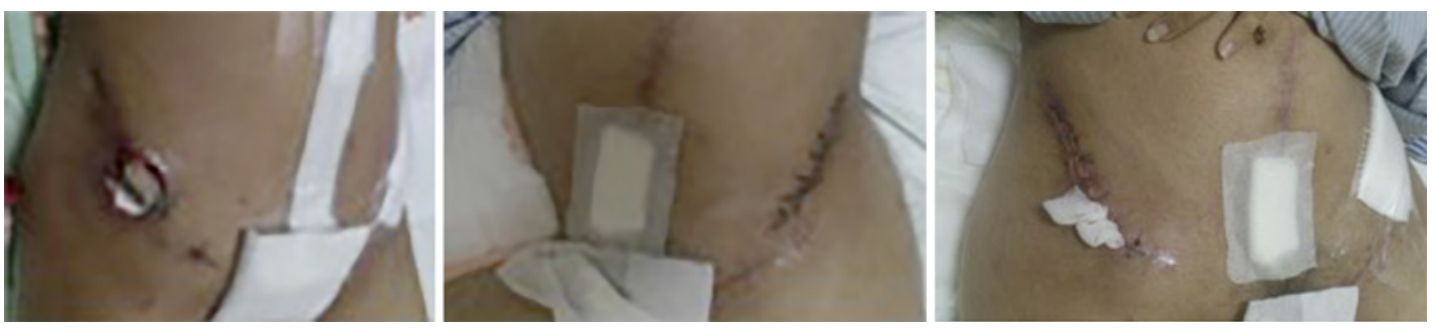

Fig. 6. Surgical site infection with bone exposed treated with VAC system. Written permission has been obtained from the patient for publication purpose. Bone exposed wound (a); dressing with VAC system (b); healing wound (c). VAC, vacuum assisted closure.

One month after surgery, there was a surgical site infection with bone exposed which upon meticulous treatment for the past 3 weeks still persisted despite intravenous antibiotics administration from the first surgery. We decided to debride and do the iliac wing osteotomy then further treated the wound with vacuum-assisted closure system for every 7-8 $\mathrm{h}$ with 80 $\mathrm{mm} \mathrm{Hg}$ vacuum-assisted closure pressure (Fig. 6). Pressure ulcer of bilateral gluteal regions was continuously treated using modern dressing (Fig. 7).

After 2 months of continuous postoperative wound care and rehabilitations, patient was finally able to sit in balance, with pelvic functional outcome Majeed score of 54 , she was finally discharged home. Patient received a total of 28 days of intravenous antibiotics and 2 weeks of oral antibiotics.

Five months after surgery, patient was allowed partial weight bearing with double crutches and upon pelvic radiograph examination, there was no loosening of the screws, pelvic ring integrity was still achieved suggesting fracture union (Fig. 8). Bilateral ankle-footorthoses and regular ankle stretching improved the patient's ankle dorsiflexion.

\section{Discussions}

Pelvic ring injuries and acetabular fractures, or combination of both, resulting from highenergy trauma are often displaced to such an extent that surgery is inevitable due to the most common injury mechanism producing these fractures, and disruptions are motor vehicle accidents in a high-energy trauma affecting many other associated injuries [3]. Such in the 
Case Reports in Orthopedic Research

\begin{tabular}{l|l}
\hline Case Rep Orthop Res 2021;4:192-201 \\
\hline DOI: 10.1159/000515563 & $\begin{array}{l}\text { @ } 2021 \text { The Author(s). Published by S. Karger AG, Basel } \\
\text { www.karger.com/cio }\end{array}$ \\
\hline
\end{tabular}

Fiolin et al.: A Case Report

Fig. 7. Left gluteal pressure ulcer due to prolonged immobilization. Written permission has been obtained from the patient for publication purpose.

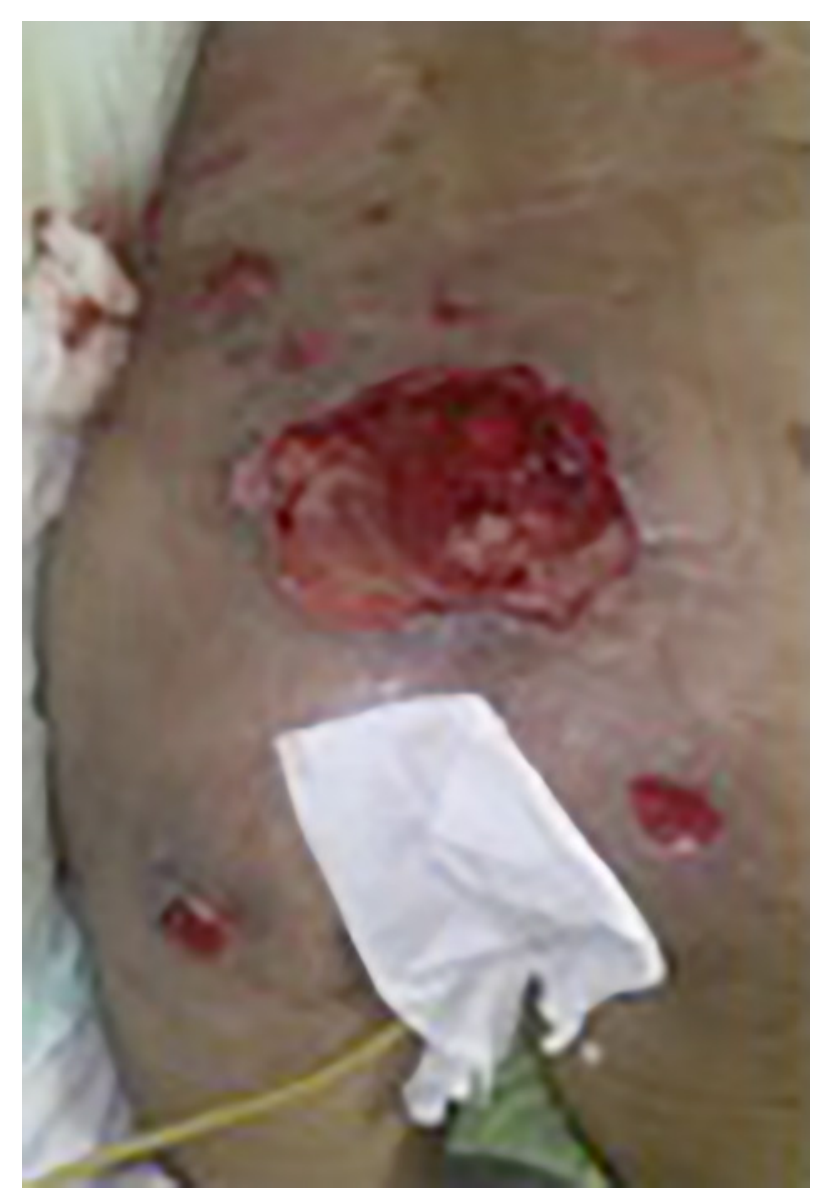

Fig. 8. Pelvic AP radiograph showing intact integrity on the pelvic-acetabular complex.

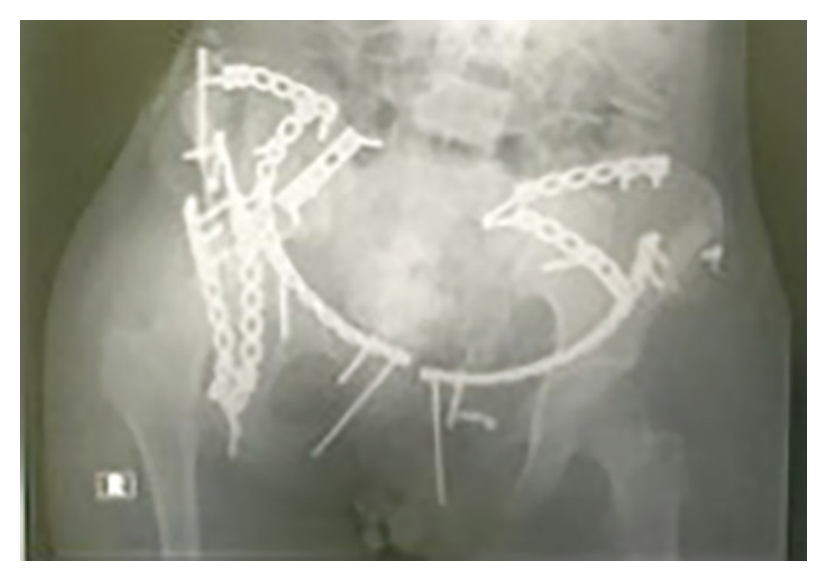

case we reported, a high-energy injury causing a massive destruction on the pelvic ring and acetabular articulation congruity with regards to the other system organ injuries.

Due to a great loss of blood volume through hemorrhage, patient was admitted to our emergency department in a severe shock state and critically ill. Primary resuscitation according to the Advanced Trauma Life Support guidelines was acknowledged. FAST scanning to determine intra-abdominal lesion was shown positive indicating a need for an emergency laparotomy in where we found an intraperitoneal bladder rupture, subserous intestinal 
laceration, and ovary lacerations, and a pelvic radiograph series was taken showing Marvin Tile C3 pelvic fracture (Young-Burgess Vertical Shear) and associated both column acetabular and coccygeal fractures. Besides primary suture of the lacerations, stable fixation of the pelvic ring was required to prevent re-lacerations and avoid the high risk of exsanguinating hemorrhage, rendering mortality in this case. Appropriate recognition and management of serious pelvic fractures are pivotal in the resuscitation strategy requiring expedited stabilization by a multidisciplinary team of trained personnel with a defined treatment protocol [4].

In patients who are both hemodynamically and mechanically unstable, and in whom the major bleeding is thought to be related to the pelvic fracture, external stabilization of the pelvis becomes the first priority. Because the main sources of bleeding are most frequently the presacral venous plexus $(80 \%)[5,6]$ and fractured bony surfaces, external stabilization decreases the hemorrhage by reducing the volume of the pelvic basin and approximating the fracture ends.

Initial pelvic radiograph series was done to evaluate the fracture fragments that showed an anteroposterior type 3 Young-Burgess pelvic fracture classification with associated both acetabular column and coccygeal fractures. Although there were a displacement and instability on the pelvic ring requiring prompt surgical management with anatomic reduction, at the current patient condition was not possible rendering a need of temporary stabilization using a less invasive, quick technique such as the anterior frame application. The anterior pelvic frame can be applied in the trauma bay, intensive care unit, or operating room in around 20-30 min.

Immediate AEF of an unstable pelvic injury has been the mainstay of acute stabilization for the past few decades. A study showed reduced mortality rates from 22 to $8 \%$ by adding acute AEF to their hospital resuscitation protocol [7]. Based on their results, they concluded that skeletal stabilization of pelvic injury should be viewed as a part of resuscitation rather than reconstruction. Burgess and colleagues [8] and others $[9,10]$ have also documented decreased transfusion needs and reduced mortality with the use of anterior external fixator. Subsequent investigators $[8,9,11-14]$ have also recommended immediate application of external fixation for hemodynamically unstable patients, and consider it a life-saving procedure. Especially in an unstable shear type, anterior external fixation application is judicious before laparotomy to reduce further bleeding and prevent decompression of the tamponade effect upon opening the abdominal wall. However, the use of anterior external fixator in sacroiliac joint disruption might aggravate the posterior instability [15].

Application of a pelvic C-clamp is another option in such sacroiliac joint disruption. Mechanically and technically, a posterior device is better than an anterior one $[14,15]$. In experienced hands, the posterior device is faster and safer to apply, and its position can be easily modified to allow access to the abdomen for the general surgeon. However, C-clamp application can not only be difficult but dangerous in cases of comminuted sacral fractures: neurovascular injury can occur due to crushing of the sacrum. In this case, patient was admitted with bilateral drop foot rendering a suspicion of sciatic nerve entrapment; hence, a C-clamp application was not performed.

Accurate early pelvic stabilization diminishes pain and hemorrhage, provides better patient nursing and comfort, and allows early mobilization. Fixation can be performed acutely, even as a component of the patient's resuscitation. Early intervention improves the likelihood of a closed reduction since the pelvic hematoma is compliant. Operative blood loss is minimal, and wound complications are unusual [7].

A definitive surgery for pelvic and acetabular fracture was carried out 6 days after the external fixation which is the "window of opportunity" in the natural history of polytrauma which allows definitive surgery on day 5-10 after initial injury. Beforehand, we evaluated the fracture fragments by a 3D CT Scan especially the involvement of weight-bearing subchondral arc of the acetabulum [4].

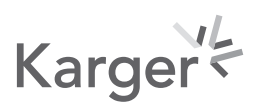


Both column fractures are defined as an acetabular fracture where no articular fragment is in connection with the axial skeleton [16]. Both column fractures are the most common pattern (19-29\%) and also the most devastating. The fracture plane travels vertically through the innominate bone, divides it anteriorly and posteriorly into at least 3 fragments. The fragments containing the acetabulum are free-floating, meaning they are no longer in continuity with the portion of the ilium that articulates with the sacrum. Therefore, the ipsilateral lower extremity is isolated from the axial skeleton and the fracture is unstable [17].

The aim of every treatment concepts in acetabular fractures is an anatomical joint restoration which leads to best clinical and radiographical results [18-20]. Acetabular fractures despite anatomic or near-anatomic reduction can potentially lead to altered stress distribution with the potential post-traumatic arthritis. The indications for surgical treatment are similar to the decision-making for simple fractures. Significant displacement of fractures extending to the weight-bearing dome of the acetabulum, incongruity of the hip joint, and hip instability generally require operative management. The majority of both column fractures are treated operatively [21]; only one-fourth of these fractures "must" be treated conservatively due to polytrauma situation, additional comorbidities, additional soft-tissue injuries, or expected stable fracture situation.

We decided to performed internal fixation to the patient to restore the pelvicacetabular congruity. The anatomic reduction remains the rationale for the surgical reduction and fixation of associated acetabular fractures and is not different from simple acetabular fracture patterns. However, the surgical approaches required, the ability to receive an anatomic reduction, and the application of rigid internal fixation techniques is more complicated. Due to the extensive involvement of both columns and pelvic ring, the use of an extensile surgical exposure to visualize and reduce these fractures has been previously recommended.

We approached the pelvis first using a right illioinguinal approach to fixate the right iliac wing, sacroiliac joint disruption, right pubic rami, and right anterior wall consecutively using a 4.5 curved reconstruction plate before further approached the left hemipelvic using a modified Stoppa with left lateral third window to fixate the left iliac wing, left sacroiliac joint disruption, left pubic rami using the same recon plate. Then finally proceed to fixate the right posterior column using Kocher-Langenbeck using a recon plate. All fixations were done under the guidance of an image intensifier to confirm adequacy of reduction and extra-articular placement of the fixation.

Similar to the study conducted by Suzuki et al. [22] recommending the use of ilioinguinal approach which allows reduction from within the pelvis by hinging fragments on their remaining capsular attachment rendering the anatomic reconstruction of anterior column possible. As stated with Hirvensalo and Lindahl [14] in a both column fractures, reduction and fixation of the anterior column should be initiated from the most cranial area. The use of anterior intrapelvic (modified Rives-Stoppa) approach for the treatment of acetabular fractures permits good to excellent reduction in the majority of cases while giving excellent visualization and access to the quadrilateral plate, and posterior column was also supported by Sagi et al. [23].

A successful surgical management in such case requires an adequate preoperative imaging and planning; choice of surgical approach, and if necessary a staged management, good quality of image intensifier and a perfect knowledge of pelvic and acetabular anatomy to prevent potentially lethal complications and allow a safe reduction and fixation.

An anatomic reduction and restoration of pelvic congruity were achieved in this patient. Postoperatively the patient had a surgical site infection on his right iliac wing with bone exposed which further underwent debridement and osteotomy of ileum to decompress the pelvic rendering wound healing. Bilateral drop foot on this patient has been existed since

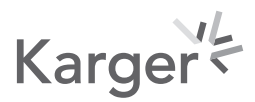


upon admission which probably due to stretched sciatic nerve due to trauma which is selflimiting. Another complication in this patient is the gluteal pressure ulcer due to prolonged immobilization of the patient initially upon admission where patient had multiple intraabdominal procedures and external fixation followed rendered the patient bedridden.

We leave the coccygeal fracture alone in this case. According to Hamoud and Abbas [24], conservative treatment of a coccygeal fracture-dislocation showed a good result due to the great potential healing and remodeling.

However, 2 months after surgery intact pelvic-acetabular congruity was still achieved confirmed with pelvic radiograph, and the patient was able to sit with aid and tried to walk using walker. Her Majeed pelvic outcome score was 54, which was considered as good enough.

A study by Matta [19] indicated that acceptable results after acetabular fracture are achieved if the femoral head is centered under the acetabular roof, is congruent and parallel, and remains in place, stable hip with no entrapment of loose bodies. Meanwhile, the predictor of operative treatment by Letournel and Judet [18] is 3 main factors of quality of reduction, interval between injury and surgery, and length of follow-up. In other words, a perfect reduction is defined as the perfect restoration of articular congruence and the reestablishment of all radiographic landmarks on 3 standard views upon the absence of postoperative CT scan evaluation in which we can observe in this case.

Until now, there has been no complete randomized trial of the management of both column fractures with pelvic fracture. However, patients who undergo operative treatment have the greatest chance for best long-term result [25].

\section{Conclusion}

Management of complex pelvic-acetabular-coccygeal fracture requires a holistic chain of treatment by emphasizing the prompt emergency management, accurate preoperative planning, and excellent execution of reconstructive surgical strategy to achieve satisfactory outcome. Excellent long-term result could be obtained by an anatomical reduction and stable fixation of pelvic-acetabulum congruity.

\section{Statement of Ethics}

Written informed consent was obtained from the patient for publication of this case report and any accompanying images.

\section{Conflict of Interest Statement}

The authors have no conflicts of interest.

\section{Funding Sources}

The authors did not receive any funding.

\section{Karger'}


Case Reports in Orthopedic Research

\begin{tabular}{l|l}
\hline Case Rep Orthop Res 2021:4:192-201 \\
\hline DOI: 10.1159/000515563 & $\begin{array}{l}\text { ○ 2021 The Author(s). Published by S. Karger AG, Basel } \\
\text { www.karger.com/cio }\end{array}$ \\
\hline
\end{tabular}

Fiolin et al.: A Case Report

\section{Author Contributions}

J.F. creates the concept, design, literature search, acquire patient data, analyses patient data, prepare, edit, and review manuscript. L.A.P.P. creates the concept, design, literature search, acquire patient data, analyses patient data, prepare, edit, and review manuscript. I.H.D. creates the concept, design, literature search, acquire patient data, analyses patient data, prepare, edit, and review manuscript.

\section{References}

1 Dalal SA, Burgess AR, Siegel JH, Young JW, Brumback RJ, Poka A, et al. Pelvic fracture in multiple trauma: classification by mechanism is key to pattern of organ injury, resuscitative requirements, and outcome. J Trauma1. 1989 Jul;29(7):981-2.

2 Olson SA, Zlowodzki M. Decision making: nonoperative and operative indications for treatment of acetabular fractures. In: Tile M, Helfet DL, Kellam JF, Vrahas M, editors. Fractures of the pelvis and acetabulum: principles and methods of management2. 4th ed. Switzerland: Thieme; 2015. p. 481-93.

3 Matta JM. Fractures of the acetabulum: accuracy of reduction and clinical results in patients managed operatively within three weeks after the injury. J Bone Joint Surg Am3. 1996 Nov;78(11):1632-45.

4 Bartlett CS III, Helfet DL. Specific surgical approaches and techniques. In: Tile M, Helfet DL, Kellam JF, Vrahas M, editors. Fractures of the pelvis and acetabulum: principles and methods of management4. 4th ed. Switzerland: Thieme; 2015. p. 507-10.

5 Heini PF, Witt J, Ganz R. The pelvic C-clamp for the emergency treatment for the unstable pelvic ring injuries: a report on clinical experience of 30 cases. Injury5. 1996;27(Suppl 1):S-A38-45.

6 Huittinen VM, Slätis P. Postmortem angiography and dissection of the hypogastric artery in pelvic fractures. Surgery6. 1973;73(3):454-62.

7 Mohanty K, Musso D, Powell JN, Kortbeek JB, Kirkpatrick AW. Emergent management of pelvic ring injuries: an update. Can J Surg7. 2005;48(1):49-56.

8 Burgess AR, Eastridge BJ, Young JW, Ellison TS, Ellison PS Jr, Poka A, et al. Pelvic ring disruptions: effective classification system and treatment protocols. J Trauma8. 1990;30(7):848-56.

9 Poka A, Libby EP. Indications and techniques for external fixation of the pelvis. Clin Orthop Relat Res9. 1996; (329):54-9.

10 Gylling SF, Ward RE, Holcroft JW, Bray TJ, Chapman MW. Immediate external fixation of unstable pelvic fractures. Am J Surg10. 1985;150(6):721-4.

11 Kellam JF. The role of external fixation in pelvic disruptions. Clin Orthop Relat Res11. 1989;241(241):66-82.

12 Trafton PG. Pelvic ring injuries. Surg Clin North Am12. 1990;70(3):655-69.

13 Wild JJ Jr, Hanson GW, Tullos HS. Unstable fractures of the pelvis treated by external fixation. J Bone Joint Surg Am13. 1982;64(7):1010-20.

14 Hirvensalo E, Lindahl J. Intrapelvic approach in acetabular fractures. In: Tile M, Helfet DL, Kellam JF, Vrahas M, editors. Fractures of the pelvis and acetabulum: principles and methods of management14. 4th ed. Switzerland: Thieme; 2015. p. 767-74.

15 Egbers HJ, Draijr F, Habemann D, Zenker W. Stabilizing the pelvic ring with the external fixator: biomechanical studies and clinical experience. Orthopade15.1992;6:363-72.

16 Matta JM. Operative indications and choice of surgical approach for fractures of the acetabulum. Tech Orthop16. 1986;1(1):13.

17 Mears DC, Velyvis JH, Chang CP. Displaced acetabular fractures managed operatively: indicators of outcome. Clin Orthop Relat Res17. 2003;407(407):173-86.

18 Letournel E, Judet R. Fractures of the pelvis and acetabulum18. Berlin, Heidelberg: Springer-Verlag; 1993.

19 Matta J. Fractures of the acetabulum: accuracy of reduction and clinical results of fractures operated within three weeks after the injury. J Bone Joint Surg Am19. 1996;78-A;1632-45.

20 Gansslen A, Hildebrand F, Krettek C. Conservative treatment of acetabular both column fractures: does the concept of secondary congruence work? Acta Chir Orthop Traumatol Cech20. 2012;79:411-5.

21 Ochs BG, Marintschev I, Hoyer H, Rolauffs B, Culemann U, Pohlemann T, et al. Changes in the treatment of acetabular fractures over 15 years: analysis of 1,266 cases treated by the German Pelvic Multicentre Study Group (DAO/DGU). Injury21. 2010;41(8):839-51.

22 Suzuki T, Smith WR, Mauffrey C, Morgan SJ. Safe surgical technique for associated acetabular fractures: a review. Patient Saf Surg22. 2013;7:1-16.

23 Sagi HC, Afsari A, Dziadosz D. The anterior intra-pelvic (modified rives-stoppa) approach for fixation of acetabular fractures. J Orthop Trauma23. 2010;24(5):263-70.

24 Hamoud K, Abbas J. Fracture dislocation of the coccyx: a case series and literature review. J Clin Case Rep24. 2017; 7:8.

25 Bircher MD. Results of treatment for fractures of the acetabulum. In: Tile M, Helfet DL, Kellam JF, Vrahas M, editors. Fractures of the pelvis and acetabulum: principles and methods of management25. 4th ed. Switzerland: Thieme; 2015. p. 927-38. 